\title{
Regulation of reproductive development by non-coding RNA in Arabidopsis: to flower or not to flower
}

\author{
Ayako Yamaguchi $\cdot$ Mitsutomo Abe
}

Received: 22 May 2012/ Accepted: 6 July 2012/Published online: 27 July 2012

(C) The Author(s) 2012. This article is published with open access at Springerlink.com

\begin{abstract}
Plants monitor environmental factors, such as temperature and day length, and also endogenous factors, such as their age and phytohormones, to decide when to flower. These cues are utilized to control expression levels of genes required for flowering. Thus, flowering time control is a unique model for understanding how gene activity is precisely regulated at the transcriptional level. In Arabidopsis, a remarkable number of non-coding RNA molecules have been identified by advanced sequencing technology. Recent progress in the flowering field has revealed several non-coding RNAs that play a major role in determining flowering time. Here, we introduce how two types of non-coding RNA species, microRNA (miRNA) and long noncoding RNA (IncRNA), contribute to flowering via regulation of target gene activity involved in this vital developmental transition.
\end{abstract}

Keywords Arabidopsis thaliana $\cdot$ Flowering $\cdot$ Long noncoding RNA (lncRNA) · MicroRNA (miRNA) · Reproductive competency $\cdot$ Vernalization

M. Abe is the recipient of the BSJ Award for Young Scientist, 2011.

A. Yamaguchi $\cdot$ M. Abe $(\square)$

Laboratory of Plant Science, Department of Biological Sciences,

Graduate School of Science, The University of Tokyo,

7-3-1, Hongo, Bunkyo-ku, Tokyo 113-0033, Japan

e-mail: mabe@biol.s.u-tokyo.ac.jp

Present Address:

A. Yamaguchi

Graduate School of Biostudies, Kyoto University,

Yoshida Konoecho, Sakyo-ku, Kyoto 606-8501, Japan

\section{Introduction}

Plants sense multiple environmental and endogenous signals to determine when to flower. For sessile plants, the ability to monitor and integrate multiple signals is essential to succeed in reproduction. Hence, a complex gene regulatory network has been constructed to enable plants to flower appropriately in time and space. Genetic and molecular analyses of flowering-time mutants in Arabidopsis has established the current model, in which five major genetically defined pathways regulate the transition from the vegetative to reproductive phase (Fig. 1; details are reviewed in Srikanth and Schmid 2011). The photoperiod pathway regulates flowering time based on day length. The vernalization pathway mediates the response to low temperature over long periods, enabling plants to sense and remember winter has come and gone. The autonomous pathway acts independently from the photoperiod and vernalization pathways, and shares a few same targets with the vernalization pathway. The gibberellin pathway defines a requirement for gibberellic acid (GA), primarily under unfavorable conditions, for flowering. The former two pathways are responsible for responding to the appropriate environmental conditions, and the latter two, reflect the endogenous status of plants.

Recently, the fifth pathway named the aging pathway, has been described by several groups (Wang et al. 2009; Wu et al. 2009; Yamaguchi et al. 2009). The importance of the endogenous status of "age" in flowering has long been recognized, since there is a reproductively incompetent stage, especially in woody plants. As plants grow, they acquire competency to respond to stimuli to produce flowers. In the case of the annual herbaceous plant Arabidopsis, there is a clear phase in which seedlings cannot respond to photoperiods (Mozley and Thomas 1995). The 


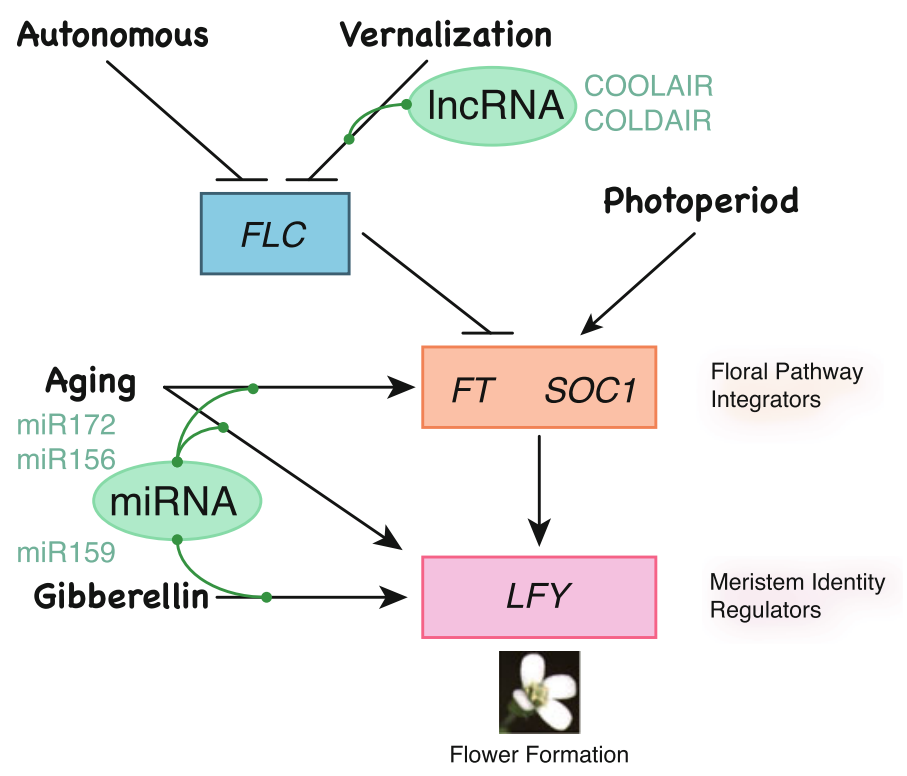

Fig. 1 Major pathways and non-coding RNAs for flowering time control. There are five major pathways controlling flowering time in Arabidopsis; autonomous, vernalization, photoperiod, aging and gibberellin pathway. The function of the autonomous and vernalization pathways is to repress the activity of FLOWERING LOCUS C $(F L C)$, a repressor of flowering, via chromatin modifications of the $F L C$ locus. The targets of repression by FLC are FLOWERING LOCUS T (FT) and SUPRESSOR OF OVEREXPRESSION OF COI (SOC1), also known as floral pathway integrators. FT and SOC1 are positively regulated by the photoperiod pathway. Environmental

aging pathway is involved in the process to acquire this reproductive competency.

These five flowering pathways together constitute a complex gene-network that exhibits crosstalk, feedbackloops and redundancies. However, this complexity as a whole can be viewed as a mechanism for the precise regulation of the activity of a relatively small number of genes, which constitute regulatory "hubs". FLOWERING LOCUS C (FLC), FLOWERING LOCUS T (FT), SUPRESSOR OF OVEREXPRESSION OF COI (SOCl) and $L E A F Y(L F Y)$ are important regulatory hubs in the control of flowering time. $F L C$ expression is regulated by the vernalization pathway and the autonomous pathway, respectively. FLC encodes a MADS-box transcription factor and acts as a dominant repressor of flowering through its control of FT and SOCl. FT and SOCl are both targeted by the photoperiod pathway at the transcriptional level as well. The activation of $F T$ is an especially crucial step in the decision to flower, since the protein encoded by $F T$ is a key component of the systemic flowering signal "florigen". After integration of environmental and endogenous inputs at the leaves via transcription of $F T$, the FT protein travels to the apical meristem where it initiates floral development (Abe et al. 2005; Wigge et al. 2005; Corbesier et al. 2007; Jaeger and Wigge 2007; Lin et al. inputs integrated by $F T$ and SOCl hubs induce expression of meristem identity regulators to start flower formation. The gibberellin pathway mainly regulates $L E A F Y(L F Y)$ expression. The recently identified aging pathway affects flowering time in two ways; first, it represses the activity of repressors of flowering, allowing plants to respond to flowering stimuli, secondly, it directly regulates the floral pathway integrators and meristem identity regulators. Two long noncoding RNAs (lncRNAs) are involved in the regulation of FLC. The aging pathway and gibberellin pathway accompany with the microRNA (miRNA) activity

2007; Mathieu et al. 2007; Notaguchi et al. 2008). The final important step to confine the floral meristem at the flanks of the apical meristem is the induction of floral meristem identity regulators, such as $L F Y$. The plant specific transcription factor LFY activates many other target genes to evoke the program for flower formation. LFY plays a role as a "hub" by integrating signals from the GA and the aging pathways, respectively.

Transcription of these regulatory hubs is tightly regulated at multiple levels. In general, tissue- and/or developmental stage-specific transcription factors can confer a specific expression profile in time and space. Regulatory information for multiple upstream transcription factors is often present on the same promoter for particular genes, which enables fine-tuned precise expression of the associated gene. At another level of regulation, chromatin modifications play an important role in regulating FLC expression (reviewed in Kim et al. 2009). Chromatin modifications are reported to regulate the levels of transcripts and/or the developmental stage specificity of expression. There are also the observations in which $F T$ and $L F Y$ expression are affected by chromatin modifiers (Adrian et al. 2011; Kinoshita et al. 2001).

Recently, a remarkable number of non-coding RNA molecules have been identified by advanced sequencing 
technologies. Intriguingly, these non-coding RNAs have received attention as another level of regulation for controlling flowering time via regulating the expression of key players. In this review, two types of non-coding RNA will be introduced; microRNAs (miRNAs) and long noncoding RNAs (lncRNAs). The function of these non-coding RNAs in the control of flowering will be described. In addition, we will discuss the future challenges in this field.

\section{MicroRNA and flowering time control}

Biogenesis and action of miRNAs in Arabidopsis

MicroRNAs are small 21-22 nucleotide (nt) RNAs that repress specific target mRNA activity at the transcriptional and/or translational level. In the early 1990s, miRNAs were discovered from the developmental timing studies in Caenorhabditis elegans. The lin-4 and let-7 miRNAs were identified as silencers of genes important for the transition from one larval stage to another (Lee et al. 1993; Reinhart et al. 2000). Not much later, multiple miRNA families were also discovered in the plant kingdom, and shown to be involved in various developmental events including flowering (Llave et al. 2002; Park et al. 2002; Reinhart et al. 2002).

The mechanism for miRNA biogenesis in plants has been well studied in Arabidopsis (Fig. 2; reviewed in Mallory et al. 2008; Xie et al. 2010). The first step of miRNA biogenesis is similar to the generation of mRNA. The primary miRNA (pri-miRNA) is transcribed by RNA polymerase II and has features in common with mRNA, including a $5^{\prime}$ cap and $3^{\prime}$ poly(A) tail. In addition, primiRNAs contain stem-loop structures that are thought to be stabilized by the nuclear RNA binding protein DAWDLE (DDL) (Yu et al. 2008). This ability to form a hairpin structure distinguishes miRNAs from other types of small RNAs.

The processing of pri-miRNAs occurs in two steps. The pri-miRNA is first cleaved into a precursor miRNA (premiRNA). The pre-miRNA is then cleaved into the mature miRNA:miRNA* duplex. DICER-LIKE 1 (DCL1), a homolog of the RNase III-like enzyme Dicer protein in animals, catalyzes the cleavage of both the pri-miRNA and the pre-miRNA. The first processing step to generate premiRNA is assisted by a dsRNA binding protein HYPONASTIC LEAVES 1 (HYL1) (Han et al. 2004; Kurihara et al. 2006; Vazquez et al. 2004) and the $\mathrm{C} 2 \mathrm{H} 2$ zinc finger protein SERRATE (SE) (Dong et al. 2008; Kurihara et al. 2006; Lobbes et al. 2006; Yang et al. 2006). After the premiRNA is further processed by DCL1 and converted into the miRNA:miRNA* duplex, the $3^{\prime}$ ends of this miRNA:miRNA* duplex are modified by the methyltransferase

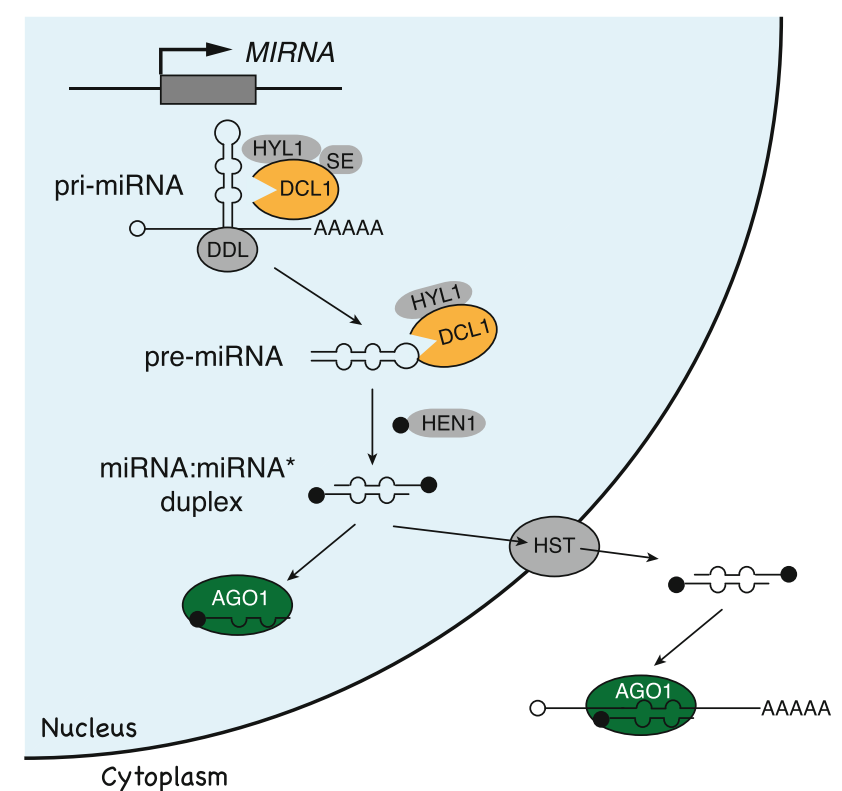

Fig. 2 miRNA biogenesis in Arabidopsis. Expression of miRNAs begins with transcription of the miRNA locus by pol II, then primiRNAs are processed through multi-steps by the RNase like enzyme DCL1 to generate the miRNA:miRNA* duplex. This duplex is modified at its $3^{\prime}$ terminus by HEN1, which prevents it from further degradation. Only the guide strands are sorted to AGO1 at the nucleus or the cytoplasm, and this leads to transcriptional or translational silencing. Refer to the text for more details

HUA ENHANCER1 (HEN1). Plant HEN1 is a nuclear protein that adds methyl groups to both strands of the miRNA:miRNA* to protect it from further modifications such as $3^{\prime}$ uridylation and subsequent degradation ( $\mathrm{Li}$ et al. 2005; Yang et al. 2006; Yu et al. 2005).

After modification by HEN1, the miRNA:miRNA* duplex is thought to be transported out from the nucleus to the cytoplasm by the function of the Exportin 5 homolog HASTY (HST), or through a HST independent mechanism (Park et al. 2005), then assembled into an RNA induced silencing complex (RISC) containing the ARGONAUTE (AGO) protein. When loading into RISC, one strand from the miRNA:miRNA* duplex is selected and stabilized. Recently, it was shown that cyclophilin 40, SQUINT (SQN), and the heat shock protein 90 (HSP90) genetically and biochemically interact with AGO1 to promote AGO activity for the loading and selecting process (Earley and Poethig 2011; Iki et al. 2011; Smith et al. 2009).

Finally, the selected strand guides RISC to the target mRNA which has complementary sequences to the miRNA. In plants, binding of a miRNA to its target mRNA mainly causes mRNA degradation triggered from a single endonucleolytic cleavage at the middle of the mRNAmiRNA duplex by the RNase $\mathrm{H}$ activity of AGO1. There are also a few examples demonstrating that miRNAs cause translational repression in plants (Yang et al. 2012), 
although this mechanism is relatively unclear compared to transcriptional silencing by miRNAs.

Role of miRNAs in flowering

Crucial functions of miRNAs in plant development are supported by the fact that mutations in genes involved in the miRNA biogenesis pathway show severe developmental defects. Bohmert et al. (1998) reported that null alleles of DCL1 and AGO1 lead to embryonic lethal phenotypes. In the case of weak alleles for these genes, the reduction of a variety of miRNAs causes pleiotropic developmental defects in leaf morphology, flowering time, flower formation and stem cell maintenance (Kidner and Martienssen 2005; Park et al. 2002; Vaucheret et al. 2004).

In Arabidopsis, there are approximately 200 miRNA families encoded by almost 300 loci (estimated by miRBase Ver18; http://www.mirbase.org/). Among numerous miRNAs, only three families so far are shown to be involved in flowering time control: $\operatorname{miR} 172$, miR156, and miR159. In the aging pathway, miR172 and miR156 play a major role and act sequentially in the process to acquire reproductive competency (Fig. 3; reviewed in Zhu and Helliwell 2011; Huijser and Schmid 2011). Expression of the miR172 family members are progressively up-regulated as plants develop in age, while miR156 levels decline as plants become older. This temporally opposite expression pattern reflects their roles in acquiring competency to respond to stimulus for flowering and promoting flowering. On the other hand, the miR159 family plays a role in the control of flowering via the gibberellin pathway (reviewed in Terzi and Simpson 2008). The mir159 miRNAs target the GAMYB family of MYB transcription factors. This family of transcription factors regulates transcription of genes induced by GA including the floral meristem identity regulator, $L F Y$. Thus, miR159 affects $L F Y$ expression and promotes flower formation through GAMYB activity. We will describe the action and function of miR172, miR156 and miR159 in more detail below.

Function of miR172 in flowering time control

The Arabidopsis genome contains five loci that generate miR172 species, MIR172a to MIR172e. Based on initial analyses of miR172 in floral patterning, miR172 is one example in which miRNAs play a role in translational repression (Chen 2004). However, recent analyses show that miR172 can also degrade target mRNA (Jung et al. 2007; Wollmann et al. 2011). In Arabidopsis, the targets of miR172 include transcripts of APETALA2 (AP2)-type genes: AP2, TARGET OF EAT1 (TOE1), TOE2, TOE3, SCHLAFMÜTZE (SMZ) and SCHNARCHZAPFEN (SNZ).

The first observation about the function of the miR172 family in flowering time control was made by Aukerman and Sakai (2003). They identified the miR172b overexpressing line named early activation tagged, dominant (eat-D) as an extremely early-flowering mutant from an activation-tagging screen. In contrast to the early-flowering phenotype of 35S:miR172, overexpression of TOE1, one of the targets of miR172, causes a late-flowering phenotype. This observation suggests that TOE1 acts as a repressor of flowering. Consistent with this, toe 1 loss-of-function mutants show a slightly early-flowering phenotype, and this phenotype is enhanced by mutations in the TOE2 gene, which is closely related to TOE1. However, toe 1 toe 2 double mutants still flower later than miR172 overexpressors, indicating the

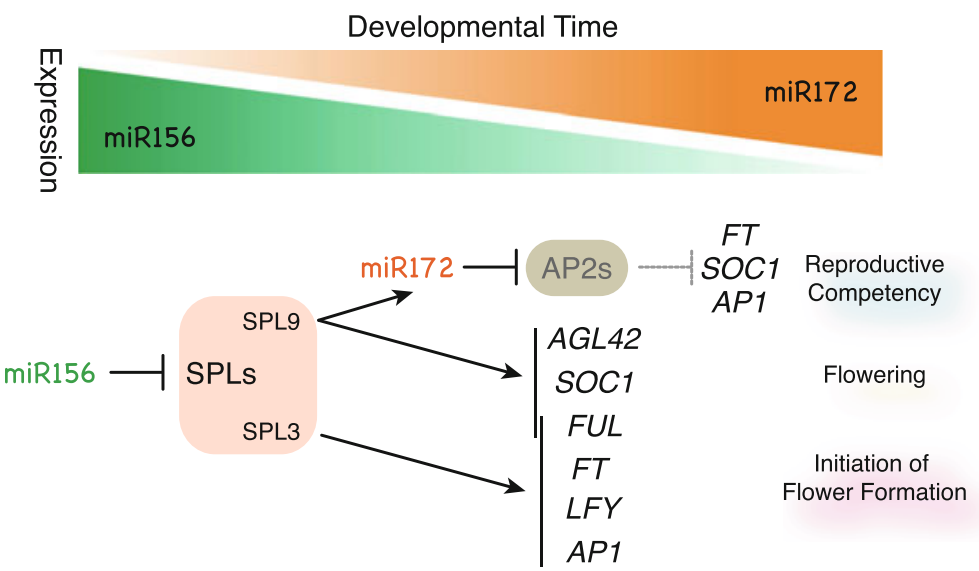

Fig. 3 The antagonistic activity of miR156 and miR172 in the aging pathway. The nature of the aging pathway depends on the sequential activity of two miRNA families. Levels of miR156 decline as plants become older. On the other hand, miR172 levels increase as plants become older. Among the 11 SPL genes targeted by miR156, SPL9 is responsible for the up-regulation of $\mathrm{miR} 172 \mathrm{~b}$, which results in reduction of AP2-type transcription factor activity, thereby enabling plants to acquire competency to respond to proper inputs from the environment to flower. In addition to miR172b, SPL9 can also directly up-regulate $S O C 1, F U L$ and $A G L 42$, a paralog of SOC1. Targets of $S P L 3 ; F U L, F T, L F Y$ and $A P 1$, are also well characterized 
existence of other factors that function redundantly with TOE1 and TOE2 to repress flowering. Recently, it was shown that $S M Z$ and $S N Z$ overexpressors flower later than wild type, and toe 1 toe $2 \mathrm{smz}$ snz quadruple mutants flower much earlier than toe 1 toe 2 double mutants, although smz snz double mutants do not affect flowering time significantly (Mathieu et al. 2009). Nonetheless, the fact that quadruple mutants still flower later than 35S:miR172 suggests further redundancy among the AP2-type genes. This is confirmed by the finding that AP2 functions as a floral repressor in addition to its well-documented roles in floral patterning. Hextuple mutants defective in all six AP2-type genes flower as early as miR172 overexpressors (Yant et al. 2010).

As mentioned above, AP2-type genes that are involved in flowering time control encode the putative transcriptional repressors. Consistent with this, AP2 can directly bind to the promoter region of the floral pathway integrator SOC1 and the meristem identity regulator APETALA1 (AP1) (Yant et al. 2010). Besides these targets, binding of the AP2 protein to the TOE3 promoter was also observed by ChIP-seq analysis (Yant et al. 2010), suggesting that there might be a feedback loop between AP2-type genes, although the significance of this loop remains unknown. Another AP2-type protein SMZ directly binds to the $1.5 \mathrm{~kb}$ downstream region of $F T$ at the leaves and seems to repress its transcription. In addition to $F T, S O C l$ and $A P l$ are also directly targeted by SMZ at the shoot meristem (Mathieu et al. 2009). Furthermore, FT is also proposed as a target of TOE1 and TOE2 proteins based on the observation that FT expression was upregulated in toe 1 toe 2 double mutants (Jung et al. 2007). The spatial expression patterns of TOE1 in the leaf vasculature also support the idea that $F T$ is a target of TOE1, although direct association of TOE1 and/or TOE2 on the FT promoter remains to be shown.

The transcription levels of AP2-type genes except TOE3 decline over time after germination (Aukerman and Sakai 2003; Jung et al. 2007; Mathieu et al. 2009). This temporal pattern of expression seems to be the result of an increase in miR172 activity. Recently, the age-dependent accumulation of miR172 was shown to primarily originate from transcriptional regulation by a SBP box transcription factor, which itself is regulated by the temporally controlled miRNA, miR156 (Wu et al. 2009). Transcriptional regulation of MIR $172 b$ by the SBP transcription factor enables plants to acquire competency to respond to flowering stimuli through repression of AP2-type gene activity.

It was originally reported that the accumulation of the mature miR172 increases with plant age, but it is not changed by photoperiod pathway mutations, such as constans (co) (Aukerman and Sakai 2003). However, day length seems to affect miR172 levels in Arabidopsis. In long-day (LD) conditions, miR172 accumulates more than in short-day (SD) conditions (Jung et al. 2007). In contrast to the results obtained from co mutants, miR172 levels are decreased in gigantea ( $\mathrm{gi}$ ) mutants that act in the photoperiod pathway (Jung et al. 2007). Although GI is proposed to act upstream of $C O$, an observed decrease of miR172 accumulation is independent from $\mathrm{CO}$ function. This fact suggests that GI can also act independently from $\mathrm{CO}$ to regulate flowering via miR172 regulation.

Ambient temperature, another environmental factor regulating flowering, seems to influence miR172 levels as well. Lee et al. (2010) examined miRNA levels from plants grown at $16{ }^{\circ} \mathrm{C}$, and identified miR172 as an ambient temperature responsive miRNA. Intriguingly, a recent report showed that the Arabidopsis RNA-binding protein FCA modulates temperature signals and regulates primiR172 processing (Jung et al. 2012b). As it will be described in more detail below, miR172 is a major component of the aging pathway and acts downstream of miR156. However, environmental conditions can also affect miR172 accumulation probably independently from miR156. This might enable plant architecture, regulated by the miR172, plastic to ambient environment during particular developmental stages.

Function of miR156 in flowering time control

In the Arabidopsis genome, there are 10 loci that encode the miR156 family, including the recently identified MIR156i and MIR156j (Breakfield et al. 2012). The miR156 family targets SBP box transcription factors (Klein et al. 1996). Seventeen family members in Arabidopsis were named SQUAMOSA PROMOTER BINDING PROTEIN LIKE 1 (SPL1) to SPL16, including two genes, $S P L 13 A$ and SPL13B that encode the same protein. Among this family, 11 members have a target site for miR156. The early-flowering phenotype of the SPL3 overexpressor was the first evidence for the involvement of $S P L$ genes in flowering time control (Cardon et al. 1999). It was also noted that SPL3 expression levels gradually increase over developmental time (Cardon et al. 1999). In addition, Schmid et al. (2003) reported that photo-induction causes upregulation of the $S P L$ genes, including $S P L 3$, in a $C O$ and $F T$-dependent manner. However, at that time, there were no available mutations in SPL3 or other SPL genes, making it difficult to evaluate their function in flowering time control.

The identification of miR156 led to the result in which miR156 overexpression reduces the level of target SPL genes and causes a late-flowering phenotype (Schwab et al. 2005; Wu and Poethig 2006). On the other hand, when miR156 activity is sequestered by an overexpressed mimicry sequence against miR156 targets, plants with elevated levels of SPL flower extremely early (Franco-Zorrilla et al. 2007). These observations clearly indicate the importance 
of the miR156/SPL regulatory module in flowering time control.

Accumulation of miR156 is high in the embryo and in the early seedling stage (Nodine and Bartel 2010; Wu and Poethig 2006), and declines as the plant grows (Schmid et al. 2003; Wang et al. 2009; Wu et al. 2009; Wu and Poethig 2006). So far, accumulation levels of miR156 were examined in several mutants and transgenic plants that exhibit flowering phenotypes (Wang et al. 2009). However, none of these mutations affect miR156 levels. Furthermore, the plant hormones, gibberellin and auxin, also have no effect on miR156 accumulation (Wang et al. 2009). Based on these observations, it is likely that miR156 levels are independent from many of the factors that affect flowering time, instead, miR156 levels are mostly dependent on plant age.

In addition to $S P L 3$, most of the other miR156-targeted$S P L$ genes also show a gradual increase in their expression level during development (Cardon et al. 1999; Schmid et al. 2003; Wang et al. 2008, 2009; Wu et al. 2009; Wu and Poethig 2006; Yamaguchi et al. 2009). This temporal expression pattern is a reflection of miR156 activity and is important for normal development in terms of flowering time. When the regulation by miR 156 is disturbed by mutations at the miR156 target sites in SPL genes, plants can flower earlier than normal with elevated levels of SPL expression (Gandikota et al. 2007; Wang et al. 2008, 2009; $\mathrm{Wu}$ and Poethig 2006). This early flowering phenotype is consistent with the original finding made with the SPL3 overexpressor, which lacked the $3^{\prime}$ UTR containing the miR156 target site (Cardon et al. 1999).

The identification of direct targets of $S P L$ genes has revealed the molecular details of how the miR156/SPL module is involved in the control of flowering time. The miR156/SPL module appears to regulate flowering time in two different ways; first, by eliminating the repressive state for flowering produced by AP2-type gene activity via regulation of miR172: second, by directly promoting floral pathway integrators and meristem identity regulators. SPL9 was demonstrated to directly bind to the regulatory region of MIR172b and induce its expression (Wu et al. 2009). As mentioned in the former section, miR172 represses the floral repressor AP2-type transcription factors, allowing plants to respond to the proper stimuli to induce flowering. It is intriguing that the sequential action of miRNAs is also observed in the regulation of developmental timing in C. elegans (Lee et al. 1993; Reinhart et al. 2000). In addition to miR172, the floral pathway integrator SOC1 and its paralog AGL42 were identified as direct targets of SPL9 (Wang et al. 2009). Although the role of FUL in flowering time has been underestimated, $F U L$ is regulated by SPL9 and SPL3 and is responsible for the early-flowering phenotypes of the SPL9 and SPL3 overexpressors
(Wang et al. 2009; Yamaguchi et al. 2009). Furthermore, SPL3 protein directly regulates the meristem identity regulators $L F Y, A P 1$ and FUL (Yamaguchi et al. 2009). In addition, direct interaction of SPL3 on the promoter region of $F T$ was shown recently (Kim et al. 2012). In this report, $F T$ is proposed as a major output of the miR156/SPL3 module, which regulates the ambient temperature responsive flowering pathway.

Although the temporal accumulation pattern of miR156 is key in the aging pathway, the mechanism by which this temporal pattern originates from remains completely elusive and is an interesting challenge for future study. The decline in the miR156 accumulation levels originates at least from decreased activity at the transcriptional level, since pri-miR156a expression decreases over time (Wang et al. 2009). Interestingly, it was reported that if leaves already formed are defoliated, miR156 levels do not decrease (Yang et al. 2011). This observation indicates that leaves are the source of the signal(s) regulating miR156 accumulation. In addition, it was shown recently that ambient temperature affects the accumulation of miR156 and miR172 (Kim et al. 2012; Lee et al. 2010), suggesting that environmental factors can modulate plant developmental stages via affecting accumulation of these two miRNAs.

The temporal pattern of $S P L$ accumulation might also be an important step to understanding the aging pathway and the complex interaction between age and environmental inputs. As described earlier, photo-induction can induce SPL gene expression dependent on $C O$ or $F T$ activity without affecting miR156 levels (Schmid et al. 2003; Wang et al. 2009). Based on recent reports, this induction seems to be directly regulated by SOC1 and FD (Jung et al. 2012a). The interaction between the aging pathway and the photoperiod pathway may not be simple, and needs to be further examined.

Function of miR159 in flowering time control

The third miRNA species, miR159, plays a role in the gibberellin pathway. In Arabidopsis, the effect of GA on flowering was underestimated since the GA deficient mutant gal does not show a significant flowering phenotype in LD conditions. On the other hand, when grown under SD conditions, the gal mutant requires GA for flowering (Wilson et al. 1992). However, recent identification of the GA receptor, GIBBERELLIC INSENSITIVE DWARF1 (GID1), allowed a role for GA in flowering time to be reconsidered. Triple mutants of the three functionally redundant copies of the GID1 receptor flower late even under LD conditions (Griffiths et al. 2006; Willige et al. 2007). This suggests that GA acts as an important cue in flowering time control under LD conditions. 
One of the molecular targets of the gibberellin pathway has long been proposed to be $L F Y$. GA signals are mediated via the GAMYB binding elements on the $L F Y$ promoter and the application of GA elevates the promoter activity of $L F Y$ even in SD (Blazquez et al. 1998; Blazquez and Weigel 2000). The fact that a member of the Arabidopsis GAMYBs, MYB33, binds to the GAMYB binding motif of the $L F Y$ promoter in vitro and its expression can respond to an increase of endogenous levels of GA or exogenous applications of GA suggests that GAMYBs play an essential role in the GA-mediated promotion of flowering (Gocal et al. 2001).

In Arabidopsis, MIR159a, MIR159b and MIR159c comprise the miR159 family. These miRNAs mainly target the GAMYB-type transcription factors, MYB33, MYB65, and MYB101, which are potential homologues of the barley GAMYBs. A recent study has shown that $M Y B 33$ expression is strongly repressed by miR159 in vegetative tissues (Alonso-Peral et al. 2010). Furthermore, when miR159 is overexpressed, plants flower late in SD conditions with decreased levels of MYB33 and LFY (Achard et al. 2004). These observations at least suggest that miR159 is involved in flowering time control in part via the gibberellin pathway. However, the relationship between miR159 levels and GA is not as clear as expected, since miR159 levels are eliminated in GA biosynthesis mutants and exogenous applications of GA induce miR159 levels in a DELLA protein dependent manner (Achard et al. 2004). Further investigations are required to elucidate the precise interaction between miR159 and GA.

Future challenges; what should we learn next?

As described earlier, mutations in miRNA biogenesis components cause pleiotropic developmental defects by affecting accumulation of multiple miRNA families, indicating that modification of miRNA biogenesis activity usually causes a broad impact on the entire profile of miRNA accumulation. Therefore, accumulation of a particular miRNA is likely primarily controlled through transcriptional regulation of its pri-miRNA. Although transcriptional regulation of miR172 has been studied and SPL9 was identified as a direct upstream regulator, it remains elusive how transcription of the three miRNA families discussed here are regulated. Given their important function in determining flowering time, it is essential to understand how these miRNAs are regulated by age, photoperiod, ambient temperature, and GA, respectively. In general, the transcription of miRNAs appears to be regulated in a manner similar to the regulation of mRNAs, by cis-regulatory elements and trans-acting factors. Interestingly, genomic approaches suggest that several transcription factors, well known as a developmental regulators, target the miRNA promoters. The binding motifs of LFY, Auxin response factors (ARFs) and AtMYC2 were found more frequently in the promoters of miRNAs compared with the promoters of protein coding loci (Megraw et al. 2006). It might be useful to further validate these predictions in an experimental context.

Studies to understand the spatial pattern of miRNA activity from the transcription of their pri-miRNAs to repression of their targets are also important. Although multiple loci encode one particular miRNA family, only a few loci are responsible for accumulation of the mature miRNA; for example, MIR $172 b$ for the temporal accumulation of the mature miR172. We need to focus on such loci to analyze when and where their promoters are activated. In addition, the spatial pattern of mature miRNA accumulation and activity needs to be analyzed at high resolution as done in Nodine and Bartel (Nodine and Bartel 2010), given the fact that miRNAs can function as non-cell autonomous signaling molecules (Carlsbecker et al. 2010; Miyashima et al. 2011). Recently, the cell type specific miRNA expression became available for the root, revealing that miR156 is enriched in the cortex and epidermis (Breakfield et al. 2012). Accumulation of miR156 in the phloem has also been reported (Buhtz et al. 2010). It is tempting to speculate a long-range action of specific miRNAs involved in flowering time control.

Genome information is now available from a variety of species, making it possible to answer the questions regarding the evolutionary origins of miRNAs. The miR156 and miR172 families are widely conserved in angiosperms, and play a role in the regulation of developmental timing in several plants such as maize (Chuck et al. 2007). The accumulation of miR156 and miR172 could be good markers to estimate the developmental stage of different species. Such a tool might be especially useful in woody plants (Wang et al. 2011). Although the presence of miR172 has been confirmed only in angiosperms, miR156 has been identified in moss (Arazi et al. 2005), suggesting an ancient origin of the miR156/SPL regulatory module. It will be interesting to determine the ancient role of the miR156/SPL module and how this module evolved to regulate flowering time in modern-day plants.

\section{Long non-coding RNA and flowering}

Action of lncRNA in regulation of gene expression

LncRNAs are defined as RNA transcripts more than 200 nucleotides in length that lack protein-coding capability. These IncRNAs may be located within the nucleus or cytosol, and may or may not have a poly(A) tail. They are often transcribed from either strand of a protein-coding 
locus, but also arise from intergenic regions. LncRNAs are the least well-understood among various RNA species and have been called the "dark matter" of the genome. However, recent studies with advanced sequencing technology illuminate the importance of lncRNAs in transcriptional control (reviewed in Nagano and Fraser 2011; Wang and Chang 2011). Although our knowledge is still incomplete, lncRNAs appear to influence gene expression mainly in two ways, through direct effects on transcription and recruitment of chromatin modifiers. These two prototypes are not mutually exclusive.

Many examples of direct effects of transcription have come from studies in yeast. Transcription of the lncRNA SRG1 through the promoter of the adjacent SER3 gene interferes with the transcription of SER3 (Martens et al. 2004). Antisense transcripts originated from adjacent promoters can also function as enhancers of sense transcription (Camblong et al. 2007). A study of glucose starvation in yeast proposed that transcription of IncRNAs elevates the accessibility of protein-coding genes to RNA polymerases (Hirota et al. 2008). Examples in which lncRNAs directly affect transcription are also found in mammals. LncRNAs transcribed from the upstream of the human dihydrofolate reductase $D H F R$ gene forms a IncRNA-DNA complex with promoter sequences and directly interacts with the general transcription factor IIB (TFIIB), which inhibits assembly of the preinitiation complex at the promoter (Martianov et al. 2007).

LncRNAs can also cause epigenetic silencing by acting as scaffolds for the recruitment of chromatin modifiers. A well studied example is the mammalian lncRNA, Xist RNA, that is crucial for $\mathrm{X}$ chromosome inactivation (Fig. 4a, above). Xist RNA associates with the X chromosome that is inactivated. This RNA "coat" is the first model in which lncRNAs act in epigenetic silencing. Initiation of Xist transcription seems to be dependent on another IncRNA, RepA RNA, which arises from the $5^{\prime}$ end of Xist (Zhao et al. 2008). RepA recruits Polycomb repressing complex 2 (PRC2) and causes trimethylation of $\mathrm{H} 3 \mathrm{~K} 27$ on the X chromosome, resulting in inactivation of the $\mathrm{X}$ chromosome. LncRNAs can also guide chromatin modifiers to distantly located genes (in trans). For example, the HOTAIR IncRNA, which is expressed from an intergenic region of the HoxC cluster, is involved in the silencing of the HoxD cluster located on a different chromosome (Fig. 4a, below). Although the guidance mechanism is not completely understood yet, it has been shown that HOTAIR can bind to the PRC2 complex and the LSD1 complex (Tsai et al. 2010). The function of lncRNAs as scaffolds for histone modifiers might be an important component of epigenetic maintenance of cell identity across cell divisions. a

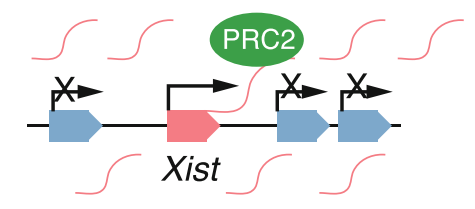

cis

trans

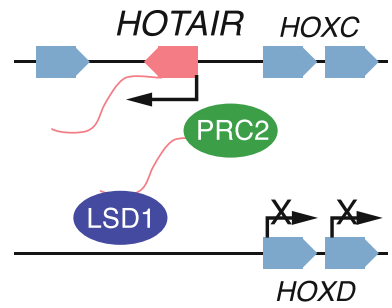

b
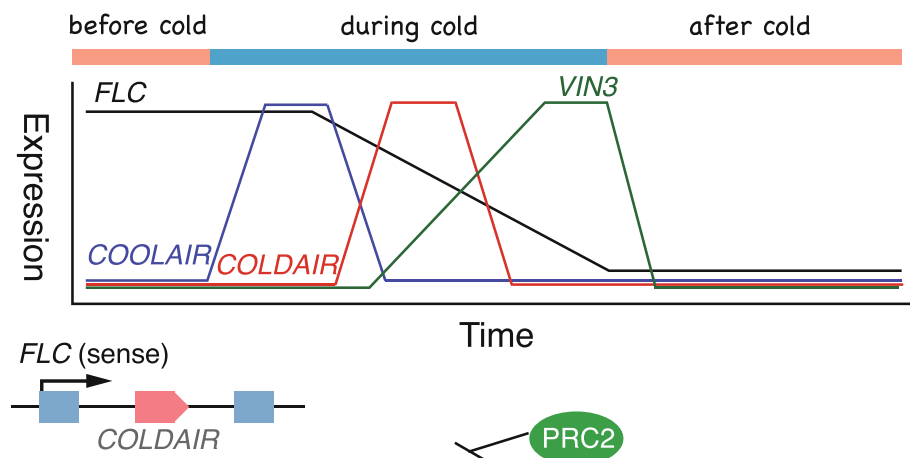

Time

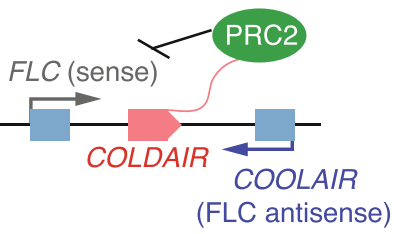

$F L C$ (sense)

$x \rightarrow$ PRC2
Fig. 4 Regulation of gene expression by lncRNA. a Recruiting chromatin modifiers by lncRNAs. Xist RNA is expressed from the inactive $\mathrm{X}$ and establishes a nuclear domain for gene silencing via directly or indirectly recruiting the histone modifier complex, the Polycomb repressor complex 2 (PRC2) (above). HOTAIR RNA is transcribed from the HOXC cluster and interacts with PRC2, which results in the methylation and silencing of several genes in the HOXD cluster in trans (below). b Regulation of $F L C$ by lncRNA. Before cold exposure, FLC is actively transcribed, at the onset of cold temperature, the FLC antisense transcript COOLAIR, accumulates. Next, COLDAIR, another lncRNA from the first intron of $F L C$, is transcribed, and represses $F L C$ sense transcripts via recruiting the PRC2 complex. Following COLDAIR accumulation, VIN3 expression is gradually increased depending on the length of cold exposure. Once back to normal temperature, VIN3 levels return to the same level before the cold. This transient expression of VIN3 is also a key to the initial repression of FLC. The repression of $F L C$ sense transcripts is maintained by activity of PRC2 after returning to warm temperature 
Action of lncRNA for epigenetic regulation on FLC

Recently, a similar function for lncRNAs in chromatin modification is also found in the regulation of $F L C$ expression, one of the key steps in Arabidopsis flowering time control (Fig. 1; reviewed in De Lucia and Dean 2011; Kim and Sung 2012a, b). High levels of FLC transcripts confer the vernalization requirement to many natural variants of Arabidopsis from northern Europe. Prolonged exposure of cold in winter reduces $F L C$ expression, and this repressive state is maintained after returning to warmer temperatures in the spring. Dissecting the mechanisms underlying the transcriptional regulation of $F L C$ has shed light on the basic principles of epigenetic silencing in plants, and now gives insight into the function of lncRNAs.

Chromatin modifications at the FLC locus before vernalization are characteristic of actively transcribed chromatin, consistent with the high level of FLC expression before cold treatment. High levels of H3K4 trimethylation are observed before cold. Complex proteins associated with the Set1 (COMPASS) complex containing H3K4 methyltransferase is responsible for adding this "active" mark on FLC chromatin, and this mark is recognized by the RNA polymerase II-associated factor 1 (PAF1) complex for active transcription. During cold, a dramatic change in chromatin status from "active" to "repressive" occurs at the FLC locus, accompanied by an increase in $\mathrm{H} 3 \mathrm{~K} 9$ and H3K27 methylation. Three key players act in this process: VERNALIZATION INSENSITIVE 3 (VIN3), VERNALIZATION 1 (VRN1) and VRN2 (Gendall et al. 2001; Levy et al. 2002; Sung and Amasino 2004). VIN3 encodes a PHD domain protein and its expression is gradually induced by exposure to cold (Fig. 4b above). Reduction of FLC does not occur in vin 3 mutants, suggesting the essential role of VIN3 for establishing the repressive status of the FLC locus. Supporting this idea, the VIN3 protein is reported to interact with the PRC2 protein (Wood et al. 2006), although how this interaction affects histone modifications needs to be addressed. The VRN1 and VRN2 protein maintain the H3K9 and H3K27 methylation caused by VIN3 at the FLC locus. The VRN2 protein shows similarity to the suppressor of zeste $(\operatorname{Su}(\mathrm{z}) 12)$ protein, which is a component of the PRC2 complex. VRN1 encodes a plant specific DNA binding protein and acts in an alternative PRC1 like complex in plants.

LncRNAs add another regulatory layer in the control of FLC transcription. Recently, the rapid accumulation of $F L C$ antisense transcripts were found as one of the earliest events in the vernalization process (Swiezewski et al. 2009). This antisense transcript named as COLD INDUCED LONG ANTISENSE INTRAGENIC RNA (COOLAIR) accumulates earlier than VIN3 induction (Fig. 4b, above; Liu et al. 2010). The COOLAIR promoter is cold inducible and can induce cold-dependent silencing of the heterologous reporter when added to the end of the reporter. From these findings, COOLAIR is proposed to silence the sense transcript of $F L C$ by promoting PRC2 recruitment. Although the function of COOLAIR in the vernalization process should be validated carefully since a contradictory result has also been reported (Helliwell et al. 2011), there is an intriguing characteristic of the COOLAIR transcript. It has two polyadenylation sites at the distal and proximal region respectively. COOLAIR polyadenylated at the distal $3^{\prime}$ end is associated with high expression of FLC. Alternatively, polyadenylation at the proximal $3^{\prime}$ end is associated with low expression of FLC. Two autonomous pathway genes, $F C A$ and $F P A$, encoding the RNA binding proteins, promote $3^{\prime}$ processing at the proximal polyadenylation site of the COOLAIR transcript, resulting in H3K4 demethylation of FLC (Hornyik et al. 2010; Liu et al. 2010). This causes down-regulation of $F L C$ transcription and promotes flowering. Although the importance of $3^{\prime}$ processing in lncRNA function has not been described in other organisms yet, it is tempting to speculate it as a common mechanism.

More recently, the second lncRNA named COLD ASSISTED INTRONIC NONCODING RNA (COLDAIR) was identified as a regulator for epigenetic silencing of FLC (Fig. 4b; Heo and Sung 2011). COLDAIR is a $1.1 \mathrm{~kb}$ transcript from the first intron of $F L C$ in the sense direction with a $5^{\prime}$ capped end but without a $3^{\prime}$ poly(A) tail, which is similar to transcripts produced by RNA polymerase $\mathrm{V}$ (PolV). However, mutations in PolIV or PolV subunits does not affect COLDAIR accumulation induced by cold. On the other hand, PolII occupancy is transiently increased by cold, suggesting that PolII is responsible for the transcription of COLDAIR. Intriguingly, CURLY LEAF (CLF), the H3K27 trimethyltransferase in the PRC2 complex of plants can bind to COLDAIR lncRNA. The authors also showed that reduced COLDAIR transcripts by RNAi compromises the vernalization response. These observations point to a similarity in the action of COLDAIR lncRNA and HOTAIR lncRNA as scaffolds for histone modifiers. It will be interesting to further explore the role of COLDAIR in recruitment and maintenance of the PRC2 association with the FLC locus.

Future challenge; how is transcription of IncRNAs regulated?

Two lncRNAs identified from the studies of epigenetic regulation of $F L C$ are both clearly induced by exposure to cold, marking the very beginning of the vernalization process. This induction is even earlier than VIN3, which is thought to be a key determinant of long exposure to cold (Fig. 4b). Although lncRNAs have long been thought of as 
transcriptional "noise", after advances in RNA sequencing technology, it is now clear that many lncRNAs show celltype specific expression and response to diverse stimuli, suggesting lncRNAs function as molecular signals. This prompts us to consider the transcriptional regulation of IncRNAs. Interestingly, in the case of Xist RNA, an antisense lncRNA named Tsix has been shown to repress Xist RNA transcription in cis, suggesting the presence of another layer of regulation (Tsai et al. 2010). Studying the transcriptional regulation of the IncRNAs COOLAIR and $C O L D A I R$, is a promising research endeavor to acquire further information on the regulatory mechanism and molecular signals that are initiated during cold exposure. In addition, it will provide us clues to understand the sensing of cold temperatures, which is currently the least described step in the vernalization process.

\section{Concluding remarks}

The advancement of sequencing technology has expanded the list of non-coding RNA species, while studies of flowering time have significantly contributed to our understanding of the function and action of miRNAs and lncRNAs as described in this review. With regard to the function of lncRNAs in plants, there are few reports except for the studies of the vernalization process. The advantage of using non-coding RNA to regulate flowering time might be related to the quantitative nature of flowering time. Using RNA as a medium may facilitate quantitative control of the key transcripts in the determination of flowering time. It might be useful to perform a regulatory process quickly without protein translation. Further studies of flowering time control promise to bring us further general insights into the regulatory mechanisms of gene expression.

Acknowledgments We thank Jennifer Pastore, Yasushi Kobayashi, Cara Winter and Kazuhisa Hiraoka for helpful comments and proofreading of this manuscript. This work was supported by BRAIN (Programme for Promotion of Basic and Applied Researches for Innovations in Bio-oriented Industry) and KAKENHI (Grants-in Aid for Scientific Research to M. A.).

Open Access This article is distributed under the terms of the Creative Commons Attribution License which permits any use, distribution, and reproduction in any medium, provided the original author(s) and the source are credited.

\section{References}

Abe M, Kobayashi Y, Yamamoto S, Daimon Y, Yamaguchi A, Ikeda Y, Ichinoki H, Notaguchi M, Goto K, Araki T (2005) FD, a bZIP protein mediating signals from the floral pathway integrator FT at the shoot apex. Science 309:1052-1056
Achard P, Herr A, Baulcombe DC, Harberd NP (2004) Modulation of floral development by a gibberellin-regulated microRNA. Development 131:3357-3365

Adrian J, Farrona S, Reimer JJ, Albani MC, Coupland G, Turck F (2011) cis-Regulatory elements and chromatin state coordinately control temporal and spatial expression of FLOWERING LOCUS T in Arabidopsis. Plant Cell 22:1425-1440

Alonso-Peral MM, Li J, Li Y, Allen RS, Schnippenkoetter W, Ohms S, White RG, Millar AA (2010) The microRNA159-regulated GAMYB-like genes inhibit growth and promote programmed cell death in Arabidopsis. Plant Physiol 154:757-771

Arazi T, Talmor-Neiman M, Stav R, Riese M, Huijser P, Baulcombe DC (2005) Cloning and characterization of micro-RNAs from moss. Plant J 43:837-848

Aukerman MJ, Sakai H (2003) Regulation of flowering time and floral organ identity by a MicroRNA and its APETALA2-like target genes. Plant Cell 15:2730-2741

Blazquez MA, Weigel D (2000) Integration of floral inductive signals in Arabidopsis. Nature 404:889-892

Blazquez MA, Green R, Nilsson O, Sussman MR, Weigel D (1998) Gibberellins promote flowering of Arabidopsis by activating the LEAFY promoter. Plant Cell 10:791-800

Bohmert K, Camus I, Bellini C, Bouchez D, Caboche M, Benning C (1998) AGO1 defines a novel locus of Arabidopsis controlling leaf development. EMBO J 17:170-180

Breakfield NW, Corcoran DL, Petricka JJ, Shen J, Sae-Seaw J, RubioSomoza I, Weigel D, Ohler U, Benfey PN (2012) Highresolution experimental and computational profiling of tissuespecific known and novel miRNAs in Arabidopsis. Genome Res 22:163-176

Buhtz A, Pieritz J, Springer F, Kehr J (2010) Phloem small RNAs, nutrient stress responses, and systemic mobility. BMC Plant Biol 10:64

Camblong J, Iglesias N, Fickentscher C, Dieppois G, Stutz F (2007) Antisense RNA stabilization induces transcriptional gene silencing via histone deacetylation in S. cerevisiae. Cell 131:706-717

Cardon G, Hohmann S, Klein J, Nettesheim K, Saedler H, Huijser P (1999) Molecular characterisation of the Arabidopsis SBP-box genes. Gene 237:91-104

Carlsbecker A, Lee JY, Roberts CJ, Dettmer J, Lehesranta S, Zhou J, Lindgren O, Moreno-Risueno MA, Vaten A, Thitamadee S, Campilho A, Sebastian J, Bowman JL, Helariutta Y, Benfey PN (2010) Cell signalling by microRNA165/6 directs gene dosedependent root cell fate. Nature 465:316-321

Chen X (2004) A microRNA as a translational repressor of APETALA2 in Arabidopsis flower development. Science 303:2022-2025

Chuck G, Cigan AM, Saeteurn K, Hake S (2007) The heterochronic maize mutant Corngrass1 results from overexpression of a tandem microRNA. Nat Genet 39:544-549

Corbesier L, Vincent C, Jang S, Fornara F, Fan Q, Searle I, Giakountis A, Farrona S, Gissot L, Turnbull C, Coupland G (2007) FT protein movement contributes to long-distance signaling in floral induction of Arabidopsis. Science 316:1030-1033

De Lucia F, Dean C (2011) Long non-coding RNAs and chromatin regulation. Curr Opin Plant Biol 14:168-173

Dong Z, Han MH, Fedoroff N (2008) The RNA-binding proteins HYL1 and SE promote accurate in vitro processing of primiRNA by DCL1. Proc Natl Acad Sci USA 105:9970-9975

Earley KW, Poethig RS (2011) Binding of the cyclophilin 40 ortholog SQUINT to Hsp90 protein is required for SQUINT function in Arabidopsis. J Biol Chem 286:38184-38189

Franco-Zorrilla JM, Valli A, Todesco M, Mateos I, Puga MI, RubioSomoza I, Leyva A, Weigel D, Garcia JA, Paz-Ares J (2007) Target mimicry provides a new mechanism for regulation of microRNA activity. Nat Genet 39:1033-1037 
Gandikota M, Birkenbihl RP, Hohmann S, Cardon GH, Saedler H, Huijser P (2007) The miRNA156/157 recognition element in the 3' UTR of the Arabidopsis SBP box gene SPL3 prevents early flowering by translational inhibition in seedlings. Plant $\mathrm{J}$ 49:683-693

Gendall AR, Levy YY, Wilson A, Dean C (2001) The VERNALIZATION 2 gene mediates the epigenetic regulation of vernalization in Arabidopsis. Cell 107:525-535

Gocal GF, Sheldon CC, Gubler F, Moritz T, Bagnall DJ, MacMillan CP, Li SF, Parish RW, Dennis ES, Weigel D, King RW (2001) GAMYB-like genes, flowering, and gibberellin signaling in Arabidopsis. Plant Physiol 127:1682-1693

Griffiths J, Murase K, Rieu I, Zentella R, Zhang ZL, Powers SJ, Gong F, Phillips AL, Hedden P, Sun TP, Thomas SG (2006) Genetic characterization and functional analysis of the GID1 gibberellin receptors in Arabidopsis. Plant Cell 18:3399-3414

Han MH, Goud S, Song L, Fedoroff N (2004) The Arabidopsis double-stranded RNA-binding protein HYL1 plays a role in microRNA-mediated gene regulation. Proc Natl Acad Sci USA 101:1093-1098

Helliwell CA, Robertson M, Finnegan EJ, Buzas DM, Dennis ES (2011) Vernalization-repression of Arabidopsis FLC requires promoter sequences but not antisense transcripts. PLoS One 6:21513

Heo JB, Sung S (2011) Vernalization-mediated epigenetic silencing by a long intronic noncoding RNA. Science 331:76-79

Hirota K, Miyoshi T, Kugou K, Hoffman CS, Shibata T, Ohta K (2008) Stepwise chromatin remodelling by a cascade of transcription initiation of non-coding RNAs. Nature 456:130-134

Hornyik C, Terzi LC, Simpson GG (2010) The spen family protein FPA controls alternative cleavage and polyadenylation of RNA. Dev Cell 18:203-213

Huijser P, Schmid M (2011) The control of developmental phase transitions in plants. Development 138:4117-4129

Iki T, Yoshikawa M, Meshi T, Ishikawa M (2011) Cyclophilin 40 facilitates HSP90-mediated RISC assembly in plants. EMBO J 31:267-278

Jaeger KE, Wigge PA (2007) FT protein acts as a long-range signal in Arabidopsis. Curr Biol 17:1050-1054

Jung JH, Seo YH, Seo PJ, Reyes JL, Yun J, Chua NH, Park CM (2007) The GIGANTEA-regulated microRNA172 mediates photoperiodic flowering independent of CONSTANS in Arabidopsis. Plant Cell 19:2736-2748

Jung JH, Ju Y, Seo PJ, Lee JH, Park CM (2012a) The SOC1-SPL module integrates photoperiod and gibberellic acid signals to control flowering time in Arabidopsis. Plant J 69:577-588

Jung JH, Seo PJ, Ahn JH, Park CM (2012b) The Arabidopsis RNAbinding protein FCA regulates microRNA172 processing in thermosensory flowering. J Biol Chem 287:16007-16018

Kidner CA, Martienssen RA (2005) The role of ARGONAUTE1 (AGO1) in meristem formation and identity. Dev Biol 280:504-517

Kim DH, Sung S (2012a) Environmentally coordinated epigenetic silencing of FLC by protein and long noncoding RNA components. Curr Opin Plant Biol 15:51-56

Kim ED, Sung S (2012b) Long noncoding RNA: unveiling hidden layer of gene regulatory networks. Trends Plant Sci 17:16-21

Kim DH, Doyle MR, Sung S, Amasino RM (2009) Vernalization: winter and the timing of flowering in plants. Annu Rev Cell Dev Biol 25:277-299

Kim JJ, Lee JH, Kim W, Jung HS, Huijser P, Ahn JH (2012) The miR156-SPL3 module regulates ambient temperature-responsive flowering via FT in Arabidopsis thaliana. Plant Physiol 159:461-478

Kinoshita T, Harada JJ, Goldberg RB, Fischer RL (2001) Polycomb repression of flowering during early plant development. Proc Natl Acad Sci USA 98:14156-14161
Klein J, Saedler H, Huijser P (1996) A new family of DNA binding proteins includes putative transcriptional regulators of the Antirrhinum majus floral meristem identity gene SQUAMOSA. Mol Gen Genet 250:7-16

Kurihara Y, Takashi Y, Watanabe Y (2006) The interaction between DCL1 and HYL1 is important for efficient and precise processing of pri-miRNA in plant microRNA biogenesis. RNA 12:206-212

Lee RC, Feinbaum RL, Ambros V (1993) The C. elegans heterochronic gene lin-4 encodes small RNAs with antisense complementarity to lin-14. Cell 75:843-854

Lee H, Yoo SJ, Lee JH, Kim W, Yoo SK, Fitzgerald H, Carrington JC, Ahn JH (2010) Genetic framework for flowering-time regulation by ambient temperature-responsive miRNAs in Arabidopsis. Nucleic Acids Res 38:3081-3093

Levy YY, Mesnage S, Mylne JS, Gendall AR, Dean C (2002) Multiple roles of Arabidopsis VRN1 in vernalization and flowering time control. Science 297:243-246

Li J, Yang Z, Yu B, Liu J, Chen X (2005) Methylation protects miRNAs and siRNAs from a $3^{\prime}$-end uridylation activity in Arabidopsis. Curr Biol 15:1501-1507

Lin MK, Belanger H, Lee YJ, Varkonyi-Gasic E, Taoka K, Miura E, Xoconostle-Cazares B, Gendler K, Jorgensen RA, Phinney B, Lough TJ, Lucas WJ (2007) FLOWERING LOCUS T protein may act as the long-distance florigenic signal in the cucurbits. Plant Cell 19:1488-1506

Liu F, Marquardt S, Lister C, Swiezewski S, Dean C (2010) Targeted $3^{\prime}$ processing of antisense transcripts triggers Arabidopsis FLC chromatin silencing. Science 327:94-97

Llave C, Kasschau KD, Rector MA, Carrington JC (2002) Endogenous and silencing-associated small RNAs in plants. Plant Cell 14:1605-1619

Lobbes D, Rallapalli G, Schmidt DD, Martin C, Clarke J (2006) SERRATE: a new player on the plant microRNA scene. EMBO Rep 7:1052-1058

Mallory AC, Elmayan T, Vaucheret H (2008) MicroRNA maturation and action-the expanding roles of ARGONAUTEs. Curr Opin Plant Biol 11:560-566

Martens JA, Laprade L, Winston F (2004) Intergenic transcription is required to repress the Saccharomyces cerevisiae SER3 gene. Nature 429:571-574

Martianov I, Ramadass A, Serra Barros A, Chow N, Akoulitchev A (2007) Repression of the human dihydrofolate reductase gene by a non-coding interfering transcript. Nature 445:666-670

Mathieu J, Warthmann N, Kuttner F, Schmid M (2007) Export of FT protein from phloem companion cells is sufficient for floral induction in Arabidopsis. Curr Biol 17:1055-1060

Mathieu J, Yant LJ, Murdter F, Kuttner F, Schmid M (2009) Repression of flowering by the miR172 target SMZ. PLoS Biol 7:e1000148

Megraw M, Baev V, Rusinov V, Jensen ST, Kalantidis K, Hatzigeorgiou AG (2006) MicroRNA promoter element discovery in Arabidopsis. RNA 12:1612-1619

Miyashima S, Koi S, Hashimoto T, Nakajima K (2011) Non-cellautonomous microRNA165 acts in a dose-dependent manner to regulate multiple differentiation status in the Arabidopsis root. Development 138:2303-2313

Mozley D, Thomas B (1995) Developmental and photobiological factors affecting photoperiodic induction in Arabidopsis thaliana Heynh. Landsberg erecta. J Exp Bot 46:173-179

Nagano T, Fraser P (2011) No-nonsense functions for long noncoding RNAs. Cell 145:178-181

Nodine MD, Bartel DP (2010) MicroRNAs prevent precocious gene expression and enable pattern formation during plant embryogenesis. Genes Dev 24:2678-2692

Notaguchi M, Abe M, Kimura T, Daimon Y, Kobayashi T, Yamaguchi A, Tomita Y, Dohi K, Mori M, Araki T (2008) 
Long-distance, graft-transmissible action of Arabidopsis FLOWERING LOCUS $\mathrm{T}$ protein to promote flowering. Plant Cell Physiol 49:1645-1658

Park W, Li J, Song R, Messing J, Chen X (2002) CARPEL FACTORY, a Dicer homolog, and HEN1, a novel protein, act in microRNA metabolism in Arabidopsis thaliana. Curr Biol 12:1484-1495

Park MY, Wu G, Gonzalez-Sulser A, Vaucheret H, Poethig RS (2005) Nuclear processing and export of microRNAs in Arabidopsis. Proc Natl Acad Sci USA 102:3691-3696

Reinhart BJ, Slack FJ, Basson M, Pasquinelli AE, Bettinger JC, Rougvie AE, Horvitz HR, Ruvkun G (2000) The 21-nucleotide let-7 RNA regulates developmental timing in Caenorhabditis elegans. Nature 403:901-906

Reinhart BJ, Weinstein EG, Rhoades MW, Bartel B, Bartel DP (2002) MicroRNAs in plants. Genes Dev 16:1616-1626

Schmid M, Uhlenhaut NH, Godard F, Demar M, Bressan R, Weigel D, Lohmann JU (2003) Dissection of floral induction pathways using global expression analysis. Development 130:6001-6012

Schwab R, Palatnik JF, Riester M, Schommer C, Schmid M, Weigel D (2005) Specific effects of microRNAs on the plant transcriptome. Dev Cell 8:517-527

Smith MR, Willmann MR, Wu G, Berardini TZ, Moller B, Weijers D, Poethig RS (2009) Cyclophilin 40 is required for microRNA activity in Arabidopsis. Proc Natl Acad Sci USA 106:5424-5429

Srikanth A, Schmid M (2011) Regulation of flowering time: all roads lead to Rome. Cell Mol Life Sci 68:2013-2037

Sung S, Amasino RM (2004) Vernalization in Arabidopsis thaliana is mediated by the PHD finger protein VIN3. Nature 427:159_ 164

Swiezewski S, Liu F, Magusin A, Dean C (2009) Cold-induced silencing by long antisense transcripts of an Arabidopsis Polycomb target. Nature 462:799-802

Terzi LC, Simpson GG (2008) Regulation of flowering time by RNA processing. Curr Top Microbiol Immunol 326:201-218

Tsai MC, Manor O, Wan Y, Mosammaparast N, Wang JK, Lan F, Shi Y, Segal E, Chang HY (2010) Long noncoding RNA as modular scaffold of histone modification complexes. Science 329:689693

Vaucheret H, Vazquez F, Crete P, Bartel DP (2004) The action of ARGONAUTE1 in the miRNA pathway and its regulation by the miRNA pathway are crucial for plant development. Genes Dev 18:1187-1197

Vazquez F, Vaucheret H, Rajagopalan R, Lepers C, Gasciolli V, Mallory AC, Hilbert JL, Bartel DP, Crete P (2004) Endogenous trans-acting siRNAs regulate the accumulation of Arabidopsis mRNAs. Mol Cell 16:69-79

Wang KC, Chang HY (2011) Molecular mechanisms of long noncoding RNAs. Mol Cell 43:904-914

Wang JW, Schwab R, Czech B, Mica E, Weigel D (2008) Dual effects of miR156-targeted SPL genes and CYP78A5/KLUH on plastochron length and organ size in Arabidopsis thaliana. Plant Cell 20:1231-1243

Wang JW, Czech B, Weigel D (2009) miR156-regulated SPL transcription factors define an endogenous flowering pathway in Arabidopsis thaliana. Cell 138:738-749

Wang JW, Park MY, Wang LJ, Koo Y, Chen XY, Weigel D, Poethig RS (2011) miRNA control of vegetative phase change in trees. PLoS Genet 7:e1002012
Wigge PA, Kim MC, Jaeger KE, Busch W, Schmid M, Lohmann JU, Weigel D (2005) Integration of spatial and temporal information during floral induction in Arabidopsis. Science 309:1056-1059

Willige BC, Ghosh S, Nill C, Zourelidou M, Dohmann EM, Maier A, Schwechheimer C (2007) The DELLA domain of GA INSENSITIVE mediates the interaction with the GA INSENSITIVE DWARF1A gibberellin receptor of Arabidopsis. Plant Cell 19:1209-1220

Wilson RN, Heckman JW, Somerville CR (1992) Gibberellin is required for flowering in Arabidopsis thaliana under short days. Plant Physiol 100:403-408

Wollmann H, Mica E, Todesco M, Long JA, Weigel D (2011) On reconciling the interactions between APETALA2, miR172 and AGAMOUS with the ABC model of flower development. Development 137:3633-3642

Wood CC, Robertson M, Tanner G, Peacock WJ, Dennis ES, Helliwell CA (2006) The Arabidopsis thaliana vernalization response requires a polycomb-like protein complex that also includes VERNALIZATION INSENSITIVE 3. Proc Natl Acad Sci USA 103:14631-14636

Wu G, Poethig RS (2006) Temporal regulation of shoot development in Arabidopsis thaliana by miR156 and its target SPL3. Development 133:3539-3547

Wu G, Park MY, Conway SR, Wang JW, Weigel D, Poethig RS (2009) The sequential action of miR156 and miR172 regulates developmental timing in Arabidopsis. Cell 138:750-759

Xie Z, Khanna K, Ruan S (2010) Expression of microRNAs and its regulation in plants. Semin Cell Dev Biol 21:790-797

Yamaguchi A, Wu MF, Yang L, Wu G, Poethig RS, Wagner D (2009) The microRNA-regulated SBP-Box transcription factor SPL3 is a direct upstream activator of LEAFY, FRUITFULL, and APETALA1. Dev Cell 17:268-278

Yang L, Liu Z, Lu F, Dong A, Huang H (2006) SERRATE is a novel nuclear regulator in primary microRNA processing in Arabidopsis. Plant J 47:841-850

Yang L, Conway SR, Poethig RS (2011) Vegetative phase change is mediated by a leaf-derived signal that represses the transcription of miR156. Development 138:245-249

Yang L, Wu G, Poethig RS (2012) Mutations in the GW-repeat protein SUO reveal a developmental function for microRNAmediated translational repression in Arabidopsis. Proc Natl Acad Sci USA 109:315-320

Yant L, Mathieu J, Dinh TT, Ott F, Lanz C, Wollmann H, Chen X, Schmid M (2010) Orchestration of the floral transition and floral development in Arabidopsis by the bifunctional transcription factor APETALA2. Plant Cell 22:2156-2170

Yu B, Yang Z, Li J, Minakhina S, Yang M, Padgett RW, Steward R, Chen X (2005) Methylation as a crucial step in plant microRNA biogenesis. Science 307:932-935

Yu B, Bi L, Zheng B, Ji L, Chevalier D, Agarwal M, Ramachandran V, Li W, Lagrange T, Walker JC, Chen X (2008) The FHA domain proteins DAWDLE in Arabidopsis and SNIP1 in humans act in small RNA biogenesis. Proc Natl Acad Sci USA 105:10073-10078

Zhao J, Sun BK, Erwin JA, Song JJ, Lee JT (2008) Polycomb proteins targeted by a short repeat RNA to the mouse $\mathrm{X}$ chromosome. Science 322:750-756

Zhu QH, Helliwell CA (2011) Regulation of flowering time and floral patterning by miR172. J Exp Bot 62:487-495 